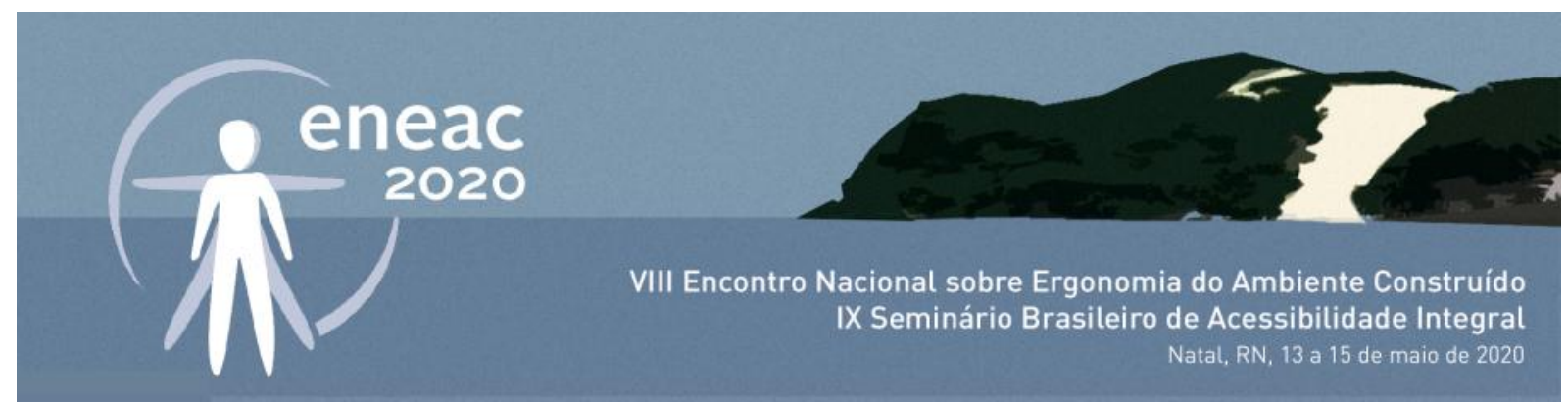

\title{
Breve panorama ergonômico do ambiente hospitalar
}

\author{
A brief ergonomic overview of the hospital environment
}

IMARA A. M. DUARTE

Doutoranda, Universidade Federal da Paraíba (UFPB), imara.duarte@gmail.com

\begin{abstract}
RESUMO
A experiência vivida em um meio hospitalar pode ser frustrante, impactante e, por muitas vezes, inseguro para pacientes, familiares e trabalhadores. Tal afirmação pode ser motivada pelas estruturas físicas dos ambientes hospitalares que por negligência projetual a algumas normas, não favorecem a percepção de segurança de seus usuários, passando a ser compreendidos como locais inseguros e estressantes. Contudo, mudanças em paradigmas culturais e sociais fizeram com que surgissem outros tipos de relações ambiente-usuário do setor de saúde, a exemplo de portais e comunidades virtuais específicos, dispositivos médicos domésticos, aumento da percepção da auto responsabilidade e do fator ambiental como gerador de saúde. Este artigo apresenta uma revisão bibliográfica e análise imagética a fim de apresentar, em um breve panorama, a apropriação da matéria ergonômica no espaço hospitalar. 0 trabalho expõe o descompasso identificado entre teoria e prática projetual em ergonomia, mostrando exemplos de fragilidades ergonômicas por meio de imagens e relatos de caso em ambientes hospitalares; em contraponto com as implicações relacionadas aos paradigmas emergentes do 'consumidor de saúde' e Telemedicina.
\end{abstract}

PALAVRAS-CHAVE: ergonomia, ambiente hospitalar, percepção, consumidor de saúde, telemedicina

\section{ABSTRACT}

The experience lived in a hospital environment can be frustrating, impactful and, often, unsafe for patients, family members and workers. Such a statement can be motivated by the physical structures of hospital environments that, due to design negligence to some standards, do not favor the perception of safety of its users, coming to be understood as unsafe and stressful places. However, changes in cultural and social paradigms have given rise to other types of environment-user relationships in the health sector, such as specific virtual portals and communities, domestic medical devices, increased perception of self-responsibility and the environmental factor as a generator of health. This article presents a bibliographic review and image analysis in order to present, in a very brief overview, the appropriation of ergonomic material in the hospital space. The article exposes the mismatch identified between theory and design practice in ergonomics, showing examples of ergonomic weaknesses through images and case reports in hospital settings; in contrast to the implications related to the emerging paradigms of the 'health consumer' and Telemedicine.

KEYWORDS: ergonomics, hospital environment, perception, health consumer, telemedicine 


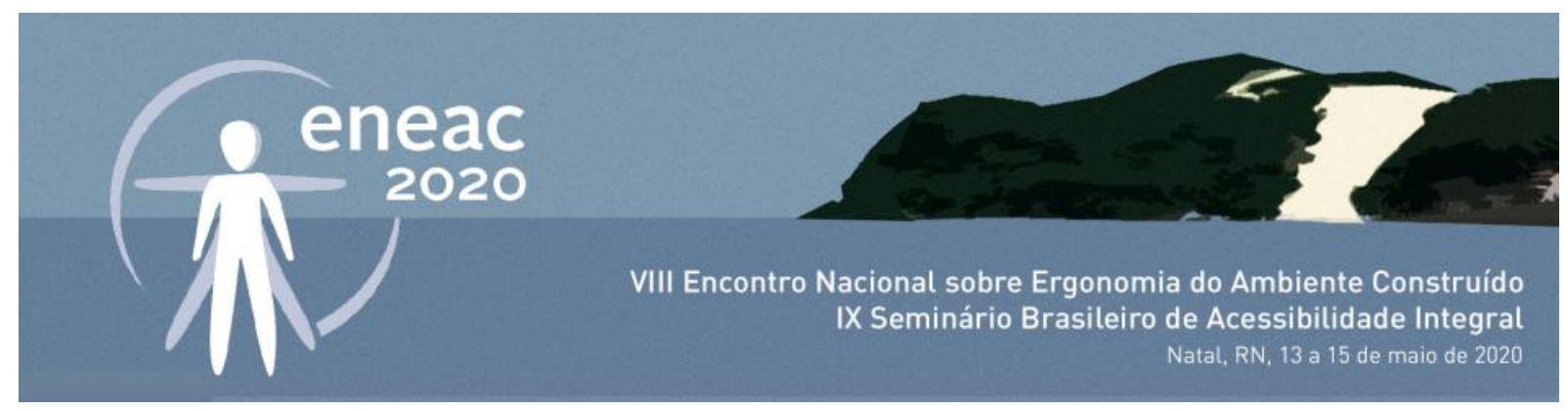

\section{INTRODUÇÃO}

A experiência vivida em um meio hospitalar pode ser frustrante, estressante e, por muitas vezes, inseguro para pacientes, familiares e trabalhadores. Mahnke (1996) afirma que, especialmente para alguns usuários, estes espaços se apresentam como um 'país estranho', com indumentárias, objetos e linguagem própria e que, por centenas de anos, foram associados à dor, morte e sofrimento. Esta pode ser motivada pelas estruturas físicas dos ambientes hospitalares que não favorecerem a percepção de segurança aos seus atores, transformando-os em locais percebidos como inseguros e estressantes. (DUARTE, 2019; IYER; STEIN; FRANKLIN, 2020).

Apesar do cenário negativo, é necessário que o ambiente hospitalar funcione em perfeito sincronismo e eficiência - técnica e funcionalmente. O design de um ambiente hospitalar deve ser desenvolvido visando à interação eficiente 'com' e 'entre' os usuários; vindo a ser ambientalmente sustentável; adequado ao clima; e que observe as necessidades tecnológicas da prática médica para a acomodação entre equipamentos, procedimentos e ocupantes. (MAHNKE, 1996; VASCONCELOS, 2004).

De acordo como Ministério da Saúde, a concepção de um ambiente hospitalar possui três princípios norteadores que visam um espaço viabilizador do processo de trabalho, confortável, com foco na privacidade do indivíduo, fazendo-o refletir em sua atividade; além de proporcionar o realce de elementos ambientais que impactam a percepção humana (como a iluminação, cor, cheiro, som, formas) de modo a garantir o bem estar a todos os usuários e; servir como ferramenta facilitadora do processo de trabalho funcional, favorecendo a otimização de recursos e o atendimento humanizado, acolhedor e resolutivo. (MINISTÉRIO DA SAÚDE, 2004).

Contudo, o conceito de ambientes com atividades relacionadas à saúde inclui, porém não se limitada as unidades hospitalares. O conceito envolve também as Unidades Básicas de Saúde (UBS), clínicas médicas, laboratórios clínicos, hospitais-dia, entre outros; abrangendo casas de repouso, centros de reabilitação, unidades de cuidados domiciliares; o que aumenta desta forma o domínio para implementação de diretrizes ergonômicas relacionadas ao setor, de acordo com várias tendências importantes na área de saúde.

Para tanto, um novo posicionamento dos projetistas está sendo exigido diante do que lyer, Stein, Franklin (2020) consideram como 'novos conceitos e situações'. Segundo os autores, o foco vai além das experiências de consumo do usuário de forma que o ambiente possa ser gerenciável e dinâmico e, de acordo com Foucault (1979/1984) ser uma ferramenta de cura. Os autores sugerem que deva ser levado em conta a

experiência e engajamento do paciente; bem-estar holístico e apoio à cura; custos crescentes e insustentáveis; envelhecimento da população e aumento da demanda; eventos adversos e riscos à segurança; escassez e esgotamento clínico; regulamentos em mudança, tendências do setor e cultura; inovação tecnológica rápida e contínua. (IYER; STEIN; FRANKLIN, 2020, p.853, tradução nossa).

A partir do exposto, o presente trabalho apresenta um breve panorama da apropriação da matéria ergonômica no espaço hospitalar; elaborado com base em revisão de literatura e análise imagética de exemplos de áreas hospitalares, que faz parte de uma pesquisa de doutorado em andamento, na Universidade Federal da Paraíba/PPGAU. A referida pesquisa de doutorado se propõe a ponderação do impacto ambiental de áreas hospitalares pouco exploradas e/ou emergentes sobre a percepção do usuário. $\mathrm{O}$ artigo expõe o descompasso identificado entre teoria e prática projetual em ergonomia, 


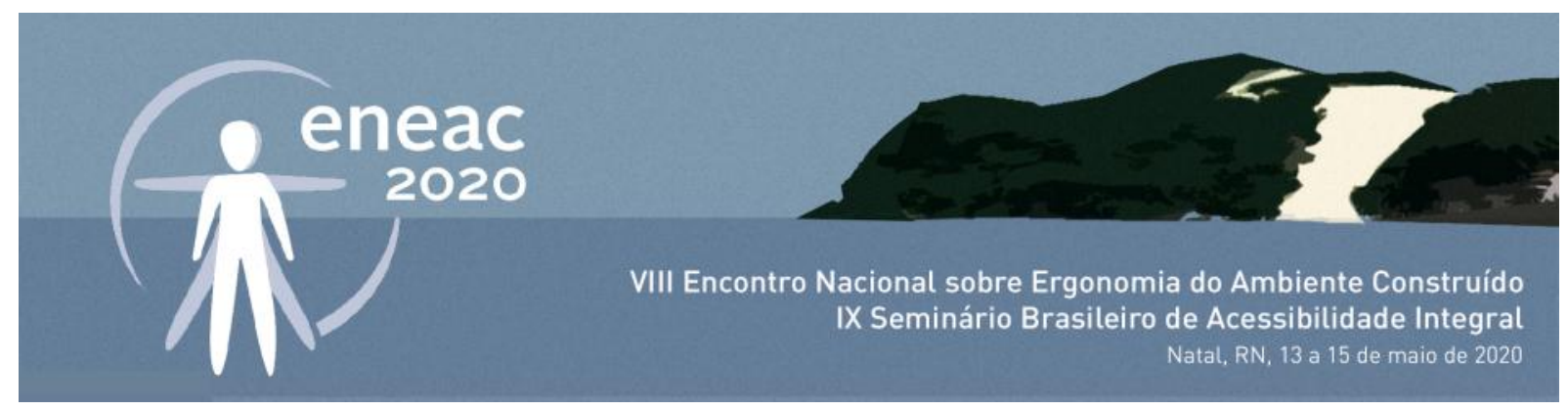

mostrando exemplos de fragilidades ergonômicas por meio de imagens e relatos de caso de ambientes hospitalares; em contraponto com as implicações relacionadas ao paradigma emergente do 'consumidor de saúde' e Telemedicina.

\section{BREVE REVISÃO E CONSIDERAÇÕES}

\section{Da ergonomia e o local de saúde}

A ergonomia é integrante do fenômeno relacional do homem com o meio que o envolve, se manifestando desde a mais remota antiguidade, de início intuitivamente, chegando a nós hoje com as mais diversas abordagens. Os primeiros entendimentos científicos desta relação vieram a ser apresentados no fim do século dezessete pelo médico Bernardino Ramazzini (1633-1714) com sua obra seminal 'De Morbis Artificum Diatriba', na qual apresenta um estudo sistemático sobre a saúde ocupacional do indivíduo em pouco mais de cinquenta atividades laborais da época. Ramazzini lançou as bases para a análise do local de trabalho como auxiliar no diagnóstico médico e como indicador de possíveis riscos para os trabalhadores. (FRANCO, 1999; SERRANHEIRA; UVA; SOUSA, 2010).

Posteriormente, de modo inovador a enfermeira Florence Nightingale (1860) lançou mão de um projeto arquitetônico diferenciado, utilizando a iluminação natural como forma de assepsia, referencial cronológico, bem como a ventilação cruzada, realojamento de lavanderias e cozinhas e redução da altura entre o teto e o piso. Estes procedimentos contribuíram para alterações do local anteriormente focado na prática médica, transformando-o em um ambiente com princípios ergonômicos, projetado para o enfermo. (MATARAZZO, 2010; DUARTE, 2019).

Nightingale (1860) ainda apresentou em seu livro seminal 'Notes On Nursing', estudos em estatística médica, saneamento, planejamento e aparência do ambiente hospitalar; dando início à consolidação de práticas existentes no setor nos dias de hoje. A visão ergonômica passou a voltar-se ao próprio ambiente de prática médica, com o foco na melhoria da interação pessoa-ambiente e em métodos mais eficazes de procedimentos sanitários.

\section{Da ergonomia do ambiente hospitalar e sua multifuncionalidade}

O ambiente hospitalar foi considerado como um "instrumento terapêutico" e como "ferramenta da tecnologia médica" para Foucault (1979/1984, pp. 99-101). O termo "máquina de curar" foi cunhado pelo autor em referência ao ambiente hospitalar, reconhecido à época como remédio eficaz capaz de dirimir potenciais agentes prejudiciais aos seus usuários. Este ambiente "medicalizado" seria, portanto, comparada a uma máquina, com design assertivo e devidamente adaptada às suas funções e aos seus usuários. (DUARTE, 2019).

Além do mais, é no setor de saúde e especificamente no ambiente hospitalar que "problemas relacionados ao ambiente de trabalho ruim podem ser prejudiciais ou fatais para o paciente, tornando o problema particularmente grave". (NEUMANN et al.,2019, p.112, tradução nossa). Serranheira, Uva e Sousa (2010) indicam que a ergonomia pode contribuir para a segurança do paciente por meio de: intervenções projetuais dos postos de trabalho, dos procedimentos, 


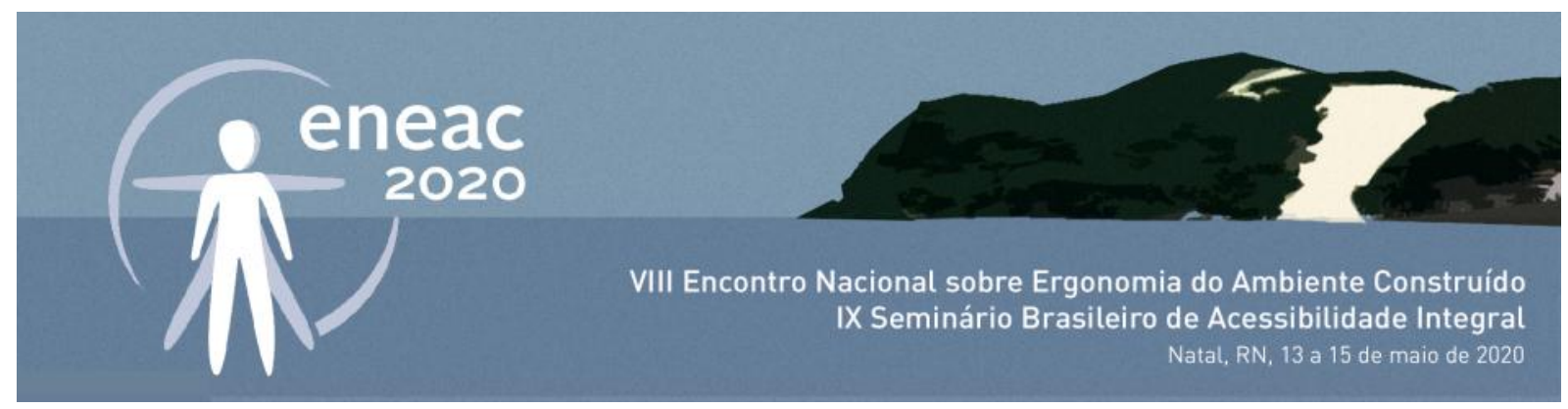

organização hierárquica, de turnos laborais, avaliação de máquinas e equipamentos, bem como fazendo parte da formação dos profissionais de saúde.

De acordo com Neumann et al. (2019) pressões excessivas e a falta de reconhecimento da dedicação individual ou da equipe, por exemplo, podem gerar stress e acarretar tensões físicas com problemas osteomusculares e visuais. Ainda, Seo e Lee (2016) afirmam que o ambiente de trabalho é uma variável importante que impacta no desempenho dos enfermeiros e que a satisfação e o 'poder de voz' são as variáveis que mediam a relação dos mesmos com o ambiente de saúde.

Relativo aos pacientes em ambientes para tratamento de saúde, Jones (2013) lança uma provocação quando questiona o que aconteceria se houvesse provas que as pessoas se recuperam melhor em locais projetados adequadamente e bem cuidados. Ele evoca o trabalho original de Ulrich (1984) que mostrou o impacto da presença de janelas (e a visão para o ambiente externo) na recuperação do paciente e afirma que há muitos indícios que a qualidade ambiental afeta a percepção sensorial, cognitiva, o que vem a impactar na segurança e eficiência das atividades ali realizadas.

A partir da pesquisa de Ulrich (1984), pesquisadores diversos a exemplo de Mahnke (1996); Melo et al. (2019); Neumann et al. (2019); Duarte (2019); Hojs, Fissell e Roy (2020) entre outros, consideram que, no caso de ambiente de saúde, os sons, a forma e a aparência geral adequados, eliciam sensações mais agradáveis e conforto relatado. Especificamente relativo à cor e iluminação; Mahnke (1996); Devlin e Arneill (2003); Dalke et al.(2006); Macallister (2016); Ghamari e Amor ( 2016); são exemplos dos que sugerem que estes elementos possam afetar diretamente o ciclo biológico circadiano dos pacientes hospitalizados, que possuem uma maior necessidade de repouso.

Ainda em relação a cor e luz como exemplo de itens ambientais de impacto sensorial; estudiosos afirmam que a ausência de iluminação natural adequada e a presença de iluminação artificial ininterrupta, com acréscimo cromático de alto valor (como pelo uso de revestimentos na cor branca ainda que de baixa intensidade de refletância) podem levar ao aumento do estresse,

comprometimento da imunidade, termo regulação interrompida e delírio; tais fatores ainda podem dificultar o processo de cicatrização, postergando assim o período de permanência no local e, por fim, contribuir para o avanço da doença (DALKE et al.,2006; MACALLISTER, 2016; GHAMARI; AMOR, 2016), mesmo que não conscientemente percebido. (ANDRADE; FERNAUD; LIMA, 2013).

Birren (1950) pondera que a má aplicação da iluminação e da cor pode levar ao cansaço visual, monotonia visual com consequente perda sensorial, desorientação e colapso do sistema nervoso e endócrino, de modo especial a pessoas sob tratamento medicamentoso. Deve haver um planejamento da iluminação em conjunto com a cor dos revestimentos, afirma César (2003, p. 187), pois, a não observância destes aspectos no planejamento luminotécnico pode causar "desconforto visual decorrente da não aplicação de princípios de comportamento da luz sobre superfícies coloridas".

Os elementos ambientais (cor, iluminação, som, cheiro, temperatura, umidade e layout) podem gerar um impacto inconsciente e profundo em seus usuários. É possível que o stress físico gerado na equipe pelo ambiente físico, "seja mascarado e mediado por outros fatores mais relevantes ou próximos, como o turno de trabalho, carga de trabalho, suporte ou outros fatores do ambiente organizacional e social" - o ambiente sociotécnico. (ANDRADE; FERNAUD; LIMA, 2013, p. 98, tradução nossa). 


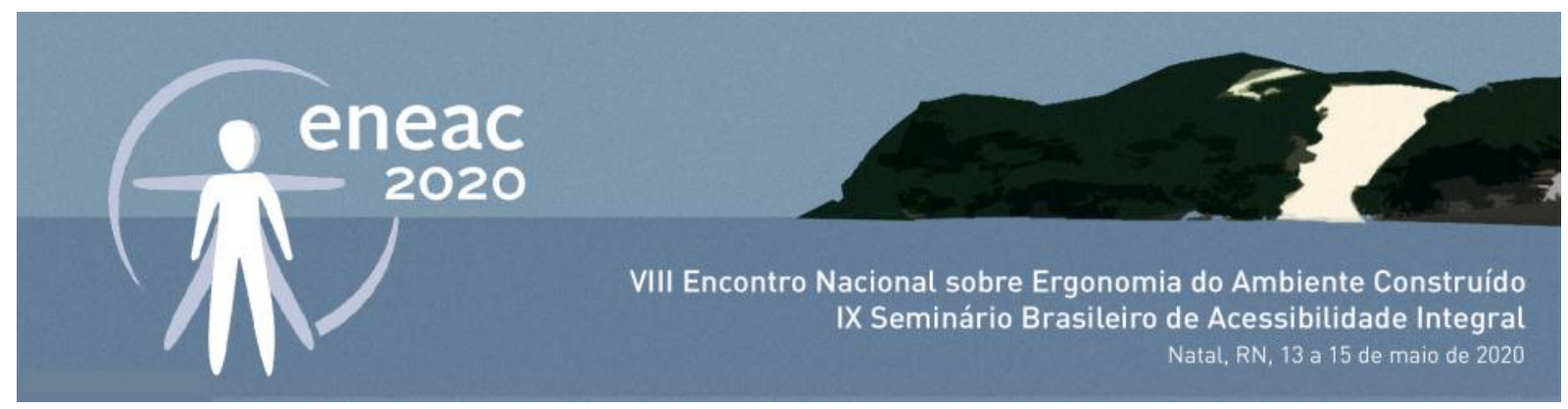

A qualidade de projetação de um sistema ergonômico sociotécnico (o qual envolve os aspectos sociais de uma organização, bem como de seus aspectos técnicos), pode ser resumida superficialmente pelo que Wise e Wise (1988) chamam de 'problema de múltiplos objetivos' (grifo nosso). A multifuncionalidade do ambiente hospitalar se verifica na medida que este assume a forma de local de trabalho, ambiente de tratamento, laboratório de experimentos e local de negócios (eventualmente) (IYER; STEIN; FRANKLIN, 2020) e, ainda, ramifica-se em diversas especialidades bem como nas especificidades dos procedimentos e equipamentos.

\section{Da regulamentação e prática}

No Brasil, Política de Humanização do Atendimento de Saúde - Humaniza SUS - do Ministério da Saúde, instrui sobre o atendimento e a estrutura física das unidades de saúde, tanto públicas quanto as particulares conveniadas. A referida política possui três focos que alicerçam sua concepção, são eles: (a) espaço que viabilize o processo de trabalho, com conforto, focado na privacidade individual, entre outros; (b) destaque para elementos ambientais que afetam o homem como a iluminação, cheiro, formas, cor, som, para garantir bem estar aos usuários; (c) agir como ferramenta viabilizadora do processo de laboral de modo a favorecer a melhoria de recursos e o atendimento humanizado, acolhedor e resolutivo. (BRASIL, 2004, 2017).

Além dos alicerces conceituais citados, a política 'Humaniza SUS' apoia uma diretriz denominada "Ambiência". Suas orientações auxiliam na projetação de espaços físicos de saúde para além do "olhar normativo, tecnicista e regulador para a produção das Ambiências". (BRASIL, 2017, p. 7). De acordo com a referida política, a "Ambiência" engloba três outras sub-diretrizes: "confortabilidade"; espaço de "encontro, produção de saúde e subjetividades"; e "ferramenta facilitadora do processo de trabalho".

Ainda assim, a Norma Regulamentadora № 17 "estabelece parâmetros que permitam a adaptação das condições de trabalho às características psicofisiológicas dos trabalhadores, de modo a proporcionar um máximo de conforto, segurança e desempenho eficiente". Nela, se incluem as condições ambientais e a organização do trabalho recomendadas, na qual destacamos iluminação, som e temperatura, dentre as demais.

Tendo como finalidade a atualização da NR17, o comitê técnico recomendou responsável em sua última assembleia, asseverou a manutenção da "essência da ergonomia". Para elaboração de recomendações específicas foi indicada a necessidade de realização de um diagnostico prévio, contudo, o entendimento é que a norma se aplica a todos os ambientes e situações laborais, cabendo à organização identificar demandas relacionadas para adaptação às características psicofisiológicas dos trabalhadores, contingenciando planos de ação específicos, além da avaliação do mobiliário das unidades de trabalho e suas condições ambientais. (BRASIL, 2019).

\section{ANÁLISE IMAGÉTICA, RELATOS DE CASO E TENDÊNCIA}

\section{Análise de imagens e relatos de caso - considerações}

Segue-se breve análise imagética de exemplos de duas unidades hospitalares (centro de hemodiálise e unidade de terapia intensiva) com a finalidade de comparar (ainda que de modo sintético) as 


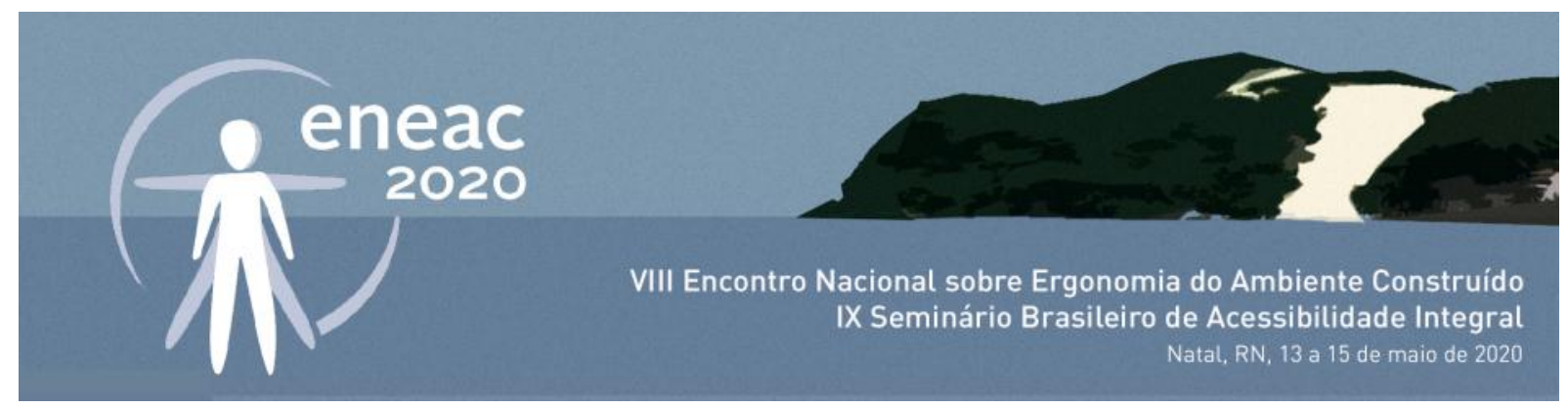

condições ambientais representadas com alguns pontos regulamentares das condições do ambiente de trabalho constantes na NR17; em função de duas características físicas, quais sejam: iluminação e cor, e o modo como foram apresentadas nos registros.

Quanto a iluminação - geral ou suplementar- a norma cita que essa deve ser "uniformemente distribuída e difusa" bem como apta a "evitar ofuscamento, reflexos incômodos, sombras e contrastes excessivos". Além disso, as diretrizes da ABNT (Associação Brasileira de Normas Técnicas), através da NBR 5413 - lluminância de interiores, no item 5.3.28 - Hospitais, complementa com informações técnicas necessárias para elaboração de projetos de iluminação em hospitais, os quais devem ser observados. Contudo, por meio de uma breve análise da imagem, é possível identificar desvio na aplicação desta normativa, como pode ser visto no exemplo da figura 1.

Figura 1. Unidade de Hemodiálise e pontos críticos referentes a NR17, na cidade de João Pessoa-PB. Onde: A - Iluminação direta e intensa; B - área de intenso contraste visual; C - Iluminação direta e intensa; D - fenômeno especular; E - onipresença da cor branca.

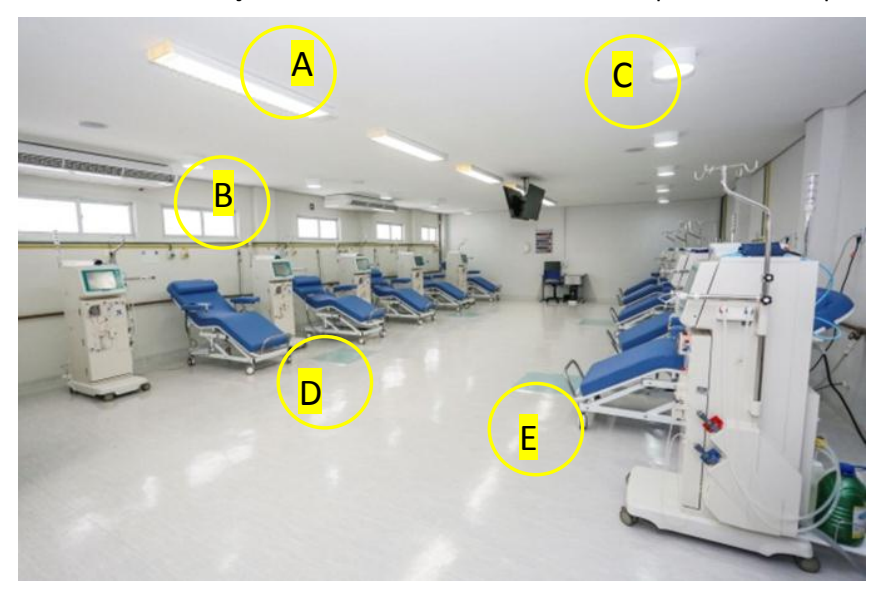

Fonte: Portal Correio (2018).

Na figura 1, observa-se que a iluminação do ambiente é forte,branca, fria, com várias áreas de sombra, televisores suspensos como ponto de interesse. Em ambientes similares, foram relatados pelos pacientes casos de percepção de luz excessiva e forte incômodo. (Duarte, 2019).

Nos itens ' $A$ ' e ' $C$ ' presença de luz branca "dura" ou direta que pode causar ofuscamento, pois produz sombras de contorno nítido, de alto contraste; item ' $\mathrm{B}$ ' apresenta alto contraste entre a iluminação externa da janela e a interna gerando necessidade de equilíbrio ocular, o que pode causar fadiga visual e náuseas; item ' $D$ ' fenômeno especular pela alta refletância da superfície na cor 'branco neve' dos pontos de iluminação, podendo levar ao deslumbramento ocular por reflexão e alterar a execução de tarefas, em especial as de precisão; item ' $E$ ' a onipresença da cor branca, de acordo com os estudos de Dijkstra, Pieterse e Pruyn (2008) é emocionalmente debilitante, capaz de ser uma fonte estressora e prejudicial se visualizada por longos períodos.

Outro caso relacionado a fragilidades de projeto ergonômico, relacionadas à luz e cor em áreas de saúde no serviço de terapia renal, detalha o aspecto do local de estudo como sendo "duas salas brancas [...] bem como uma sala amarela destinada a pacientes infectados pelo vírus da hepatite $B$ " (grifo nosso). (MELO et al., 2019, p.941). A descrição dos autores revela a negligência ergonômica no uso da cor amarela em uma sala com acometidos por hepatite, situação na qual é possível que um exame clínico seja prejudicado pela interferência da cor ambiente, a qual pode reforçar o aspecto da 


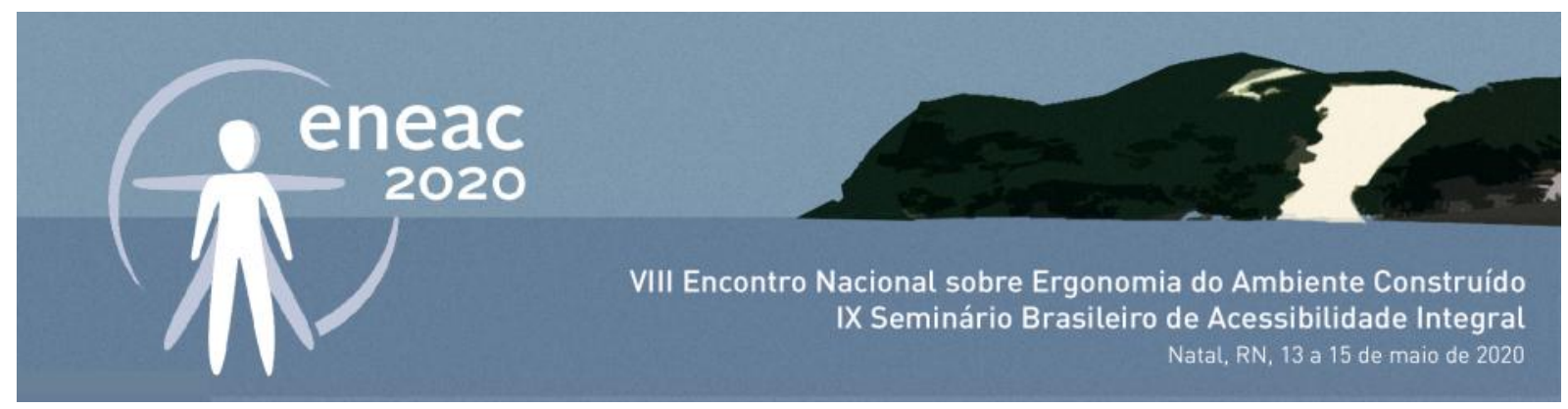

tez amarelada do paciente. (MAHNKE, 1996; DALKE et al., 2006). Segue na figura 2 outro exemplo deste tipo de falha cromática comum que impacta em pacientes acometidos com febre amarela.

Figura 2. Unidade de Terapia Intensiva para tratamento da febre amarela com máquina de hemodiálise - UTI "Ala Amarela". Hospital Márcio Cunha, Ipatinga/MG.

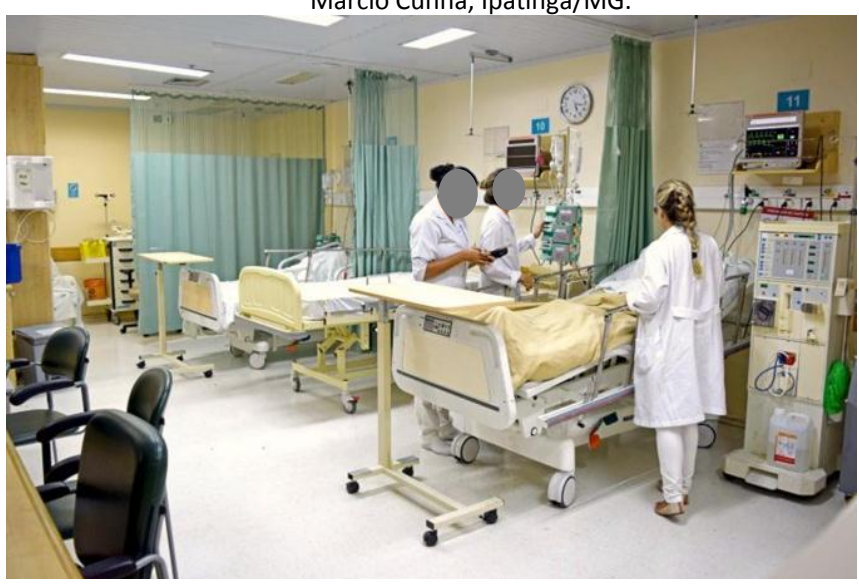

Fonte: Portal Hospitais Brasil (2017).

\section{Das considerações sobre aspectos térmicos e sonoros}

Para além da análise imagética, no que diz respeito ao aspecto térmico, a NR17 estabelece entre vinte e vinte e três graus centrígrados a temperatura recomendada para salas de cuidados de saúde. Tal indicação é na prática ignorada nos ambientes de hemodiálise (por exemplo) tornando-os sensivelmente mais frios, alegadamente em função do maquinário ou da alta temperatura externa ao ambiente. A característica ambiental 'conforto térmico' foi item reportado pelos usuários como 'afeto negativo' e 'desconforto' nos estudos de Duarte (2019) e Melo et al.(2019).

Com relação ao aspecto sonoro, a Norma Regulamentadora no 15 é a sua orientadora. Nela estão estabelecidos parâmetros para atividades e operações insalubres, e tem em seus anexos 1 e 2, respectivamente, limites de tolerância para ruídos contínuos ou intermitentes, bem como limites de tolerância para ruídos de impacto. A NR15 esclarece que o ruído intermitente ou contínuo, para os fins de aplicação de Limites de Tolerância, não seja de impacto e disponibiliza uma tabela correlacionando o tempo de exposição diária máxima possível ao nível de ruído medido em decibéis.

No caso do ruído de impacto, a norma o conceitua como apresentando picos de energia acústica com duração menor que um segundo e em intervalos maiores que um segundo; além de estabelecer um limite de tolerância de cento e trinta decibéis. Ademais, há que se questionar o quão saudável seria para o usuário experimentar o limite estabelecido para o ruído, várias vezes ao dia ou, na melhor das hipóteses, várias vezes durante a semana.

Ocorre que em ambientes hospitalares e, mais especificamente, em Unidades de Terapia Intensiva, os alarmes estão inseridos na maioria dos dispositivos e equipamentos utilizados para efetuar um sem- número de medições das funções fisiológicas continuamente, de modo a alertarem a equipe de saúde em caso de necessidade. De acordo com WUNG e SCHATZ (2018) o volume, a duração e o tipo de som emitido, afetam negativamente a prontidão dos enfermeiros, e pouco mais de dez por cento destes ignoram ou silenciam os dispositivos. 


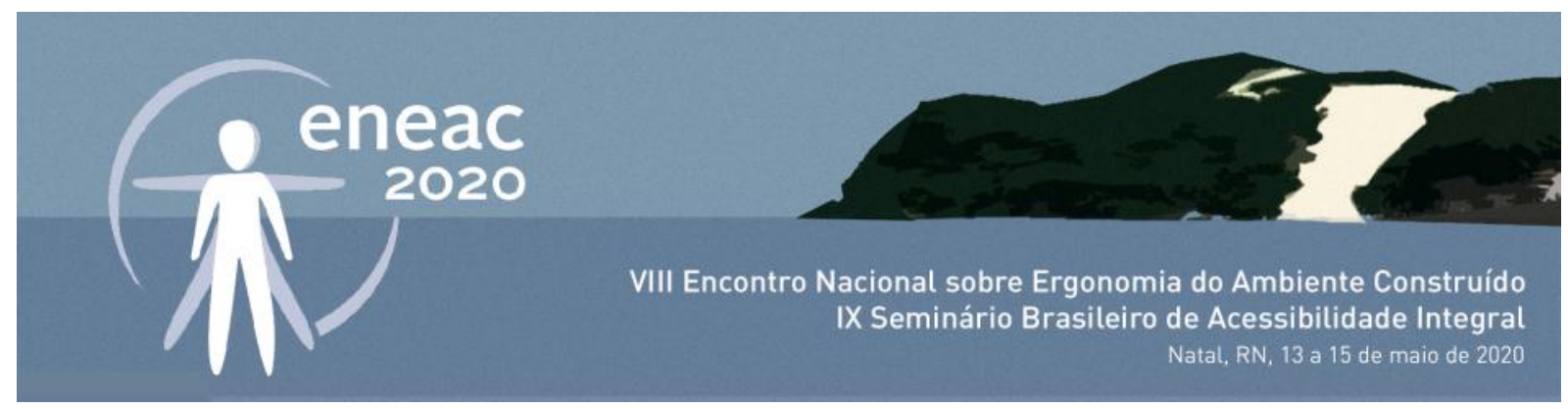

A Organização Mundial da Saúde recomenda níveis máximos de ruído durante dia e a noite para internações. Fadda (2018), no entanto, afirma que os sons das vozes e dos equipamentos do local ultrapassam em quase o dobro os valores dos limites estabelecidos, e que o ruído prevalente nas unidades está ligado a conversas da equipe, dos visitantes, e dos equipamentos.

Tais considerações se apresentam relevantes para a comparação entre os níveis de ruído efetivos e os recomendados na NR15. Em seu estudo, Feitosa (2011) afirma que ambientes de hemodiálise se equivalem ao nível de ruídos das unidades de terapia intensiva, ultrapassando "em média o nível de ruído previsto pela NBR 10.152 para ambientes de saúde (apartamentos, enfermarias, berçários, centros cirúrgicos) que é de trinta e cinco a quarenta e cinco decibéis [...]". (FEITOSA, 2011, p.32). Assim como Fadda (2018), Feitosa (2011) identificou que os ruídos mais intensos, segundo o autor, são dos alarmes das máquinas seguido do burburinho da equipe. Os ocorridos podem contribuir com aumento da sensação de desconforto do paciente e alterar o tempo de reação do profissional frente a uma emergência.

Neste aspecto, as considerações de Maran et al. (2017) e Schwabe (2017) sob a ótica do funcionamento cognitivo, avaliam que o desempenho da memória de trabalho sob o estresse dispara uma série de falsos alarmes mentais, indicando respostas imprevistas e inconsistentes, com o consequente prejuízo do uso dos affordances como meio de informação concreta para desempenho da tarefa. Além disso, o aumento no estado de ativação fisiológica (excitação, positiva ou negativa) diminui a capacidade de discriminação espaço-tempo e de aprendizagem associativa por meio do contexto, viabilizando ações de puro reflexo e com atenção reduzida, pondo em dúvida a segurança do procedimento a ser realizado.

\section{Tendência}

Até aqui foram apresentados alguns exemplos de fragilidades ergonômicas no ambiente hospitalar que ilustraram a distância entre teoria e prática; o laboratorial e o contextual; do 'voltado a atividade' ao 'voltado ao humano'. Jones (2013, p.9, tradução nossa) sintetiza tal pensamento quando o denomina de "cenários descentralizados do futuro da medicina", no qual há tentativas projetuais de articulação entre as alterações tecnológicas radicais e o significado cultural dessas mudanças.

É importante notar que, das alterações da Norma Reguladora no 15 ocorridas no ano de 2018, destaca-se a reforma no seu objetivo, ao redefinir valores conceituais, que migram do "conforto acústico em ambientes diversos" para a "preservação da saúde e do bem estar humano" (CROCE, 2017) (grifo nosso) evidenciando desta forma, o acompanhamento da tendência de aumento significativo da amplitude conceitual na abordagem ergonômica.

Diante destes cenários fragmentados, estão os três domínios de especialização da ergonomia que ora revezam-se, ora coadunam-se, quais sejam: ergonomia física (características humanas e sua relação com a atividade física); cognitiva (processos mentais, perceptivos) e; organizacional (sistemas sociotécnicos) (I.E.A, 2020). Curiosamente, foi relatado por Neumann et al. (2019) que há um desequilíbrio entre o número de estudos ergonômicos sociotécnicos e a crescente demanda para análises cognitivas dos processos de trabalho, com maior déficit na área de saúde, muito em virtude da escalada da implementação de tecnologia da informação neste setor. (JONES, 2013). 


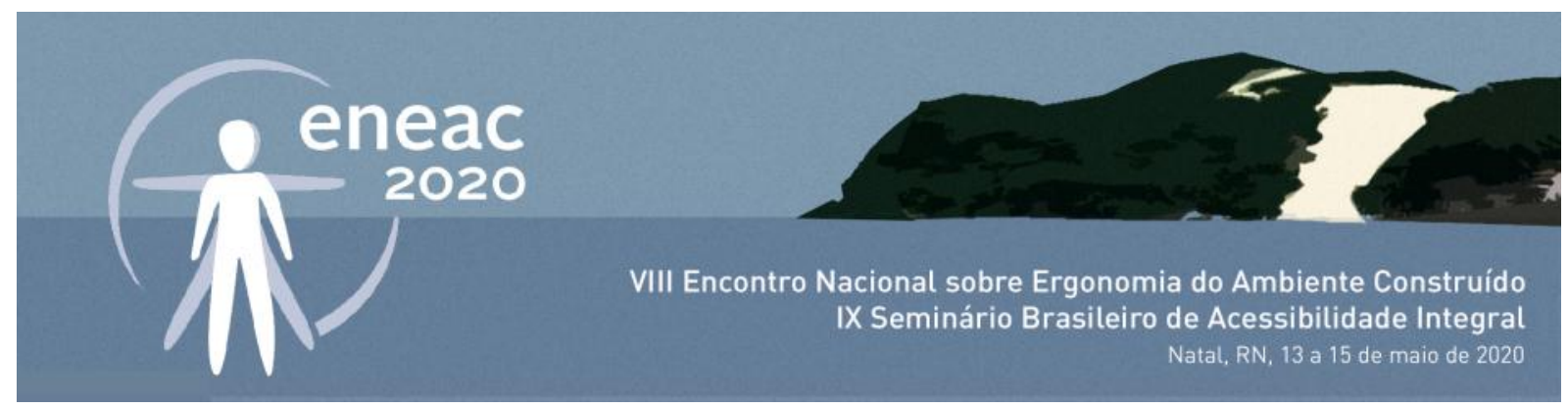

Holden, Cornet e Valdez (2020) inferem que os atores contemporâneos na assistência médica não se restringem a equipe médica, eles se expandem aos pacientes, as famílias, profissionais da área de tecnologia da informação, gestores e demais interessados que exercem atividades em prol da saúde nos mais diversos ambientes. Os autores afirmam que mudanças em paradigmas culturais e sociais fizeram com que surgissem outros tipos de relação com o setor de saúde, a exemplo de portais e comunidades virtuais específicos, dispositivos médicos domésticos; aumento da percepção da auto responsabilidade e do fator ambiental como gerador de saúde.

Segundo os autores, o paradigma emergente é o do 'consumidor de saúde'(HOLDEN; CORNET; VALDEZ, 2020). De acordo com a pesquisa, este 'consumidor' vem seguido de todo um aparato tecnológico e informacional necessários para cuidados médicos domiciliares e; para exercer o trabalho de ser 'coautor de sua própria saúde', participando ativamente nas tomadas de decisão relativas a tudo o que envolve seu bem estar.

De acordo com Toledo (2020) a Telemedicina ou Telenfermagem já está ao alcance dos usuários brasileiros a partir do ano de 2020. O termo resume em si o paradigma do 'consumidor de saúde' localizando-o em situações específicas nas quais o usuário terá acesso ao atendimento médico online, tendo como base de sua argumentação questões nas quais

A vida útil prolongada, a redução do êxodo para as grandes cidades e a maior incidência de doenças crônicas gera uma pressão de custos na saúde e uma maior dependência do suporte especializado. Neste sentido, é mais eficaz ter especialistas residentes apenas em área populacional alta, pois é necessário atender um número de pacientes que justifique o respectivo custo.

O livro recém publicado Fundamentals of Telemedicine and Telehealth, da Academic Press (2020) recomenda [...] para quase todos os pacientes, exceto emergências [...] a telemedicina tem um papel importante no apoio desta equação ao disponibilizar o melhor atendimento para todos e melhor gestão de custos, tendo sido usada com sucesso no Brasil [...].

Por exemplo, o atendimento crônico precisa de visitas mais frequentes e, portanto, é mais bem gerenciado na atenção primária - que pode e deve ser apoiado via suporte online - diminuindo a necessidade de o paciente viajar e fornecendo ao profissional uma oportunidade de aprender a gerenciar melhor futuros episódios semelhantes. (TOLEDO, 2020, p.1)

Tal paradigma encontra sérios argumentos contra sua eficácia no trabalho de Jones (2013), que propõe a observação de cada experiência vivida, objetivando a compreensão do ponto de vista das pessoas, tomando-as como pacientes e não como 'consumidores de cuidados de saúde'. $\mathrm{O}$ autor defende que o paciente não está apto - por questões emocionais e técnicas, entre outras - para tomada de decisões, como faria qualquer outro consumidor.

Todo este rápido apanhado comparativo com alguns exemplos se faz importante, pois revela o quão diverso pode ser projetar soluções ambientais ergonomicamente consistentes para o setor de saúde, uma vez que algumas bases epistemológicas estão sendo modificadas e, para além de unidade hospitalar, o ambiente a ser projetado pode ser a unidade domiciliar de cuidados de saúde ou o interior de uma unidade de saúde móvel entre outros, inclusive, atentando-se a aspectos paisagísticos.

Jones (2013) ainda argumenta que, diante desta dificuldade, é necessário lidar com a complexidade humana por meio de interações e observações, identificando limites, práticas e oportunidades diante de cada situação para que haja a transposição do design 'centrado no usuário' para o design de 'experiências significativas'. 


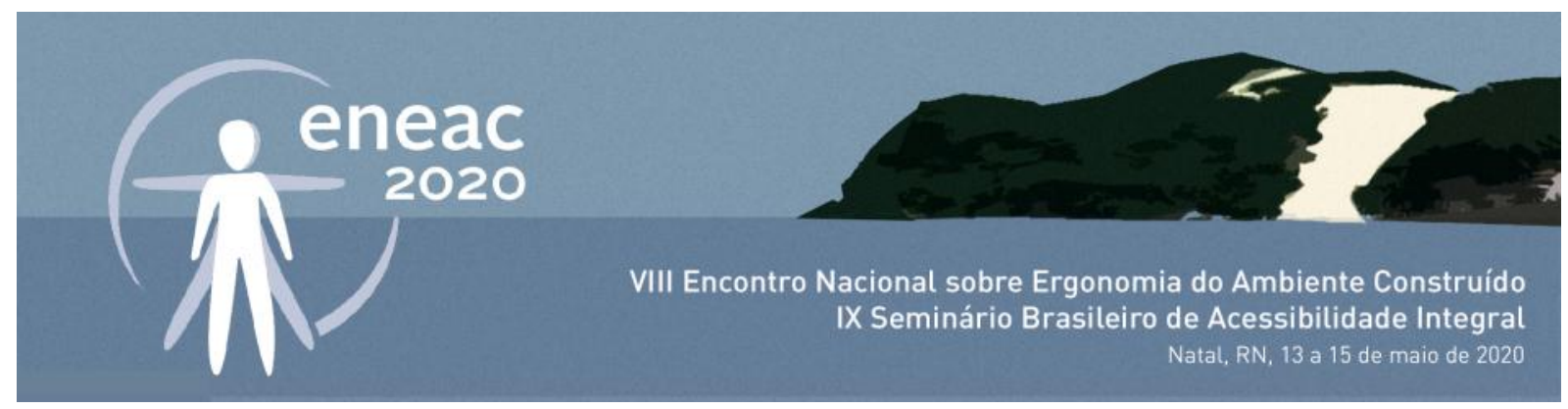

\section{CONSIDERAÇÕES FINAIS}

A despeito da provável pouca originalidade do tema e da extensa quantidade de estudos voltados à ergonomia de ambientes de saúde, é possível observar nos exemplos apresentados anteriormente o descompasso entre o avanço epistemológico das ciências da saúde e o tratamento do ambiente físico envolvido. Normas e indicações úteis e sedimentadas que remontam a recomendações dos estudos de Ramazzini (1714) e Nightingale (1860) entre outros, embora válidas hodiernamente, ainda são sistematicamente ignoradas em boa parte da realidade brasileira, em detrimento de fatores de decisão não-técnicos.

A distância entre o regulamentar e o praticado pode ocorrer - se não por falta de capacitação dos projetistas - mas pelo entendimento que certas normas são 'menores' em termos de impacto ergonômico que outras. O resultado é a fragilização de aspectos ergonômicos que deveriam ser levados em conta, mas não o foram, tornando o projeto ambiental para a área de saúde 'adoecido'.

A despeito deste 'adoecimento ambiental', algumas organizações estão adaptando modelos de prestação de cuidados em resposta às necessidades do usuário e aumentando a ênfase na experiência, bem estar e a satisfação de pacientes e profissionais. Estas têm sido identificadas como prioridades emergentes, à medida que novos paradigmas socioculturais e as tecnologias informacionais em saúde apresentam desafios para a projetação de ambientes de prestação de cuidados para além das organizações. Pode-se questionar até que ponto as normas e procedimentos ergonômicos recomendados para o setor necessitam de atualizações/adaptações, frente às mudanças de paradigmas evidenciadas pela indústria 4.0, a exemplo da telemedicina.

Cumpre destacar que, para amplificação deste brevíssimo panorama, são prementes maiores investigações, formação e/ou mobilização de grupos de estudo e trabalho transdisciplinares, de modo a sugerir modos/meios que venham a parear soluções tecnológicas emergentes com a segurança e conforto físico, psíquico e social do usuário (paciente, equipe e acompanhante); visto que a qualidade do projeto ergonômico do ambiente de saúde afeta criticamente a segurança e a eficácia deste mesmo sistema em virtude do impacto da qualidade ergonômica sobre as pessoas. (NEUMANN et al., 2019).

Ainda que tais considerações sejam aparentemente ordinárias, maiores estudos se fazem indispensáveis para o reconhecimento do impacto simultâneo de questões de ergonomia física, cognitiva e organizacional envolvendo o paciente (HOLDEN; CORNET; VALDEZ, 2020) e a medicina emergente, muito em função dos desalinhos apontados.

Diante do quadro apresentado, o presente artigo visa colaborar para que os designers, engenheiros, arquitetos e demais profissionais, entendam o impacto ambiental de seus projetos (entre eles os tecnológicos) em termos ergonômicos, levando em conta que a análise ergonômica exitosa apenas será possível por meio da cooperação entre saberes (NEUMANN et al.,2019) enquanto considerem a complexidade humana; sob pena de anacronismo funcional da disciplina.

\section{REFERÊNCIAS}

ANDRADE, C. C.; FERNAUD, E. H.; LIMA, M. L. A. better physical environment in the workplace means higher well-being? A study with healthcare professionals. Psyecology. v.4, n.1, pp.89-110. 2013.

BIRREN, F. Color psychology and color therapy. New York: McGrew-Hill Book Company Inc. 1950. 


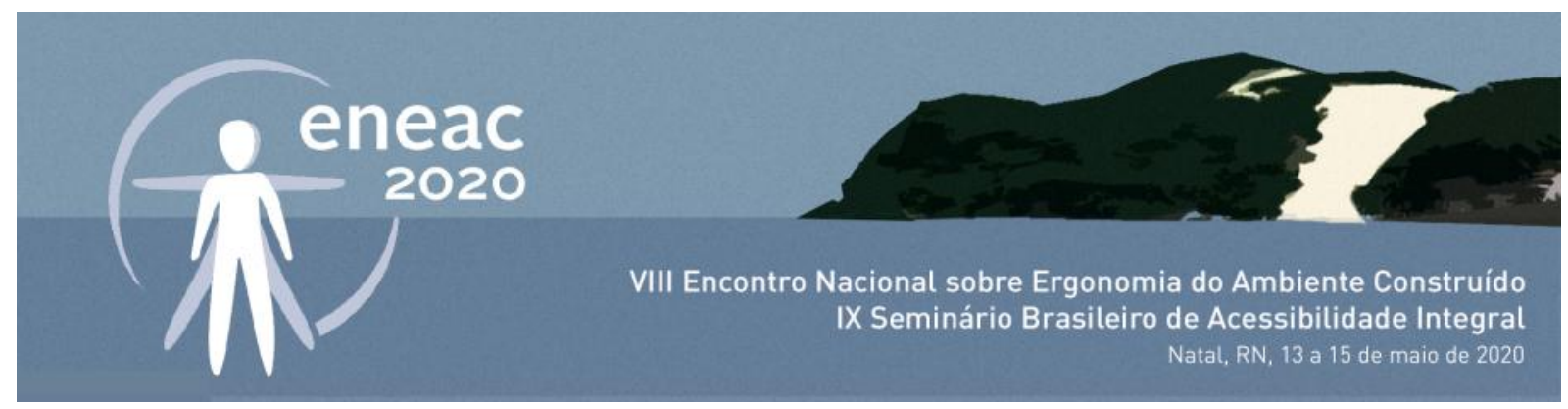

BRASIL. Ministério da Economia - Secretaria do Trabalho 2019 - Proposta para NR 17 mantém essência da ergonomia. Publicado: sexta, 13 de Setembro de 2019. Disponível na internet em http:// <trabalho.gov.br/noticias/7238-propostapara-nr-17-mantem-essencia-da-ergonomia>. Acesso em: 25 jan 2019.

. Ministério da Economia. Secretaria do trabalho. Portaria no 3.214. NR 17. Estabelece parâmetros que permitam a adaptação das condições de trabalho às características psicofisiológicas dos trabalhadores, de modo a proporcionar um máximo de conforto, segurança e desempenho eficiente. Diário Oficial da União, Brasília, 1978. Disponível na internet por em http:// por: <enit.trabalho.gov.br/portal/images/Arquivos_SST/SST_NR/NR-17.pdf>. Acesso em:25 jan 2019.

Ministério da Economia. Secretaria do trabalho. Portaria no 3.214. NR 15. Estabelece parâmetros para atividades e operações insalubres. Diário Oficial da União, Brasília, 1978. ANEXO N.ㅇ 2-LIMITES DE TOLERÂNCIA PARA RUÍDOS DE IMPACTO. 2020. Disponível na internet em http://por: <trabalho.gov.br/seguranca-e-saude-no-

trabalho/normatizacao/normas-regulamentadoras/norma-regulamentadora-n-15-atividades-e-operacoes-insalubres $>$. Acesso em:25 jan 2019.

- Ministério da Economia. Secretaria do trabalho. Portaria no 3.214. NR 15. Estabelece parâmetros para atividades e operações insalubres. Diário Oficial da União, Brasília, 1978. ANEXO N.o 1 limites de tolerância para ruídos contínuos ou intermitentes. Disponível na internet em http:// por: <trabalho.gov.br/seguranca-e-saude-no-

trabalho/normatizacao/normas-regulamentadoras/norma-regulamentadora-n-15-atividades-e-operacoes-insalubres>. Acesso em:25 jan 2019.

Ministério da Economia. Secretaria do trabalho. Portaria no 3.214, de 08 de junho de 1978. Norma Regulamentadora № 8 - Edificações - estabelece requisitos técnicos mínimos que devem ser observados nas edificações, para garantir segurança e conforto aos que nelas trabalhem. Disponível na internet em http:// por: <www.maconsultoria.com/arquivos/ea8afae9aa72bdd052f76bc20c65f9a6.pdf > Acesso em:25 jan 2019.

BRASIL, Ministério da Saúde. Secretaria de Atenção à Saúde. Política Nacional de Humanização da Atenção e Gestão do SUS. A experiência da diretriz de Ambiência da Política Nacional de Humanização - PNH / Ministério da Saúde, Secretaria de Atenção à Saúde, Política Nacional de Humanização da Atenção e Gestão do SUS. Ministério da Saúde, 44 p.: il. Brasília. 2017.

Ministério da Saúde. Secretaria-Executiva. HumanizaSUS: Política Nacional de Humanização: a humanização como eixo norteador das práticas de atenção e gestão em todas as instâncias do SUS/ Ministério da Saúde. Ministério da Saúde. Brasília. 2004.

Ministério da Saúde. Agência Nacional de Vigilância Sanitária. Resolução RDC no. 50, de 21 de fevereiro de 2002. Dispõe sobre o Regulamento Técnico para planejamento, programação, elaboração e avaliação de projetos físicos de estabelecimentos assistenciais de saúde. Disponível na internet em http:// por: <bvsms.saude.gov.br/bvs/saudelegis/anvisa/2002/rdc0050_21_02_2002.html>. Acesso em: 25 jan 2019.

CÉSAR, J. C. O. Cor e percepção ambiental: as relações arquetípicas da cor e seu uso em áreas de tratamento de saúde. 2003. 248 p. Tese (doutorado). Faculdade de Arquitetura e Urbanismo. Universidade de São Paulo. São Paulo.

CROCE, B. Como fazer um bom projeto de acústica para hospital? 3 de dezembro de 2018. Portal Acústica. Disponível na internet em http:// por: <portalacustica.info/como-fazer-um-bom-projeto-de-acustica-para-hospital/>. Acesso em:25 jan 2019.

Conforto Acústico com a NBR 10.152 - O que mudou na norma em 2017. 10 de maio de 2018. Portal Acústica. Disponível na internet em http:// por: <portalacustica.info/conforto-acustico-nbr-10-152/>. Acesso em:25 jan 2019.

DALKE, H.; et al. Colour and lighting in hospital design. Optics \& Laser Technology. v.38, Kingston. Elsevier. 2006. pp. 343365

DENHAM L. P.; BLAKEMAN, T. M.; MORRIS, R. L.; ASHCROFT, D. M. Mapping the territory of renal care: a formative analysis of the cognitive work involved in managing acute kidney injury. Ergonomics, 62:9, pp.1117-1133. 2019. DOI: 10.1080/00140139.2019.1620968.

DEVLIN, A. S.; ARNEILL, A. B. Health Care Environments and Patient Outcomes: A Review of the Literature. Environment and Behavior, v.35, Sage. 2003. p. 665. 


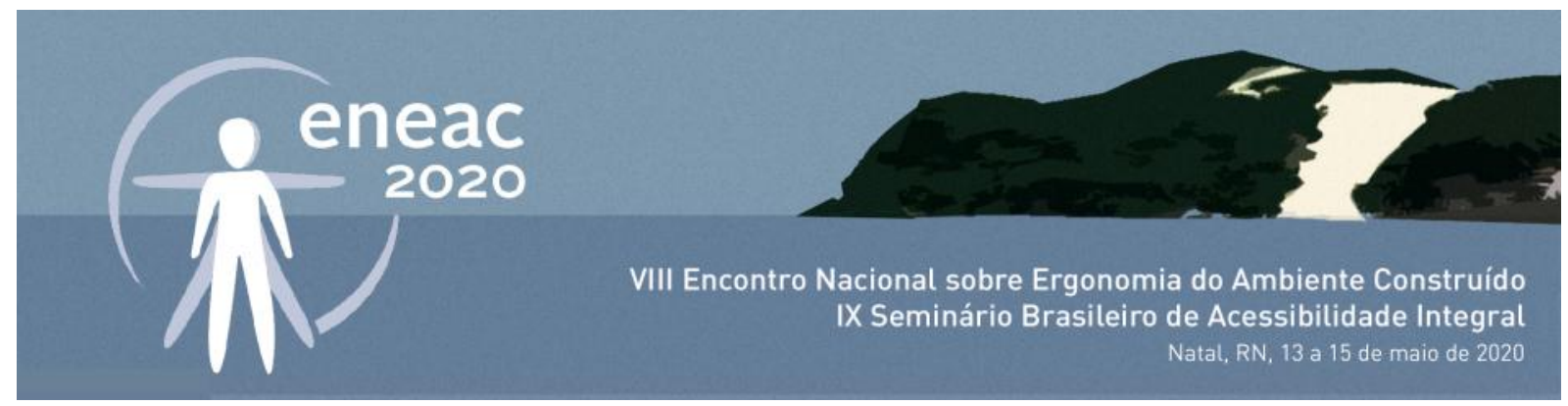

DIJKSTRA, K.; PIETERSE, M.E.; PRUYN, A.TH.H. Individual differences in reactions towards color in simulated healthcare environments: The role of stimulus screening ability. Journal of Environmental Psychology, n 28. London: Elsevier. 2008. pp.268-277.

DUARTE, I.A.M. (2019). Percepção afetiva das cores: um estudo do ambiente de hemodiálise em uso. 159p. Dissertação (mestrado). Programa de Pós Graduação em Design. Universidade Federal de Campina Grande.

FADDA, J. Quality of Healthcare: A Review of the Impact of the Hospital Physical Environment on Improving Quality of Care. Innovative Renewable Energy, 2018. pp. 217-253. DOI:10.1007/978-3-319-94595-8_20.

FEITOSA, J. M. R. (2011). Riscos no trabalho em clínicas de hemodiálise. Monografia (especialização).70p. Departamento de Engenharia Civil. Pontifícia Universidade Católica do Rio de Janeiro.

FOUCAULT, M. Microfísica do poder. (1979),4a ed. Rio de Janeiro :Edições Graal. 1984.

FRANCO, G. "Ramazzini and workers' health". Vol 354. Lancet. 1999. pp.858-61.

FREITAS, P. P. W.; COSMO, M. Atuação do Psicólogo em Hemodiálise. Revista Sociedade Brasileira de Psicologia Hospitalar SBPH .v.13,n.1, Jun. Rio de Janeiro. 2010.

GHAMARI, H.; AMOR, C. The Role of Color in Healthcare Environments, Emergent Bodies of Evidence-based Design Approach. Sociology andAnthropology. v.4, n.11, 2016. pp. 1020-1029.

HOJS , N.; FISSELL, W. H.; ROY, S. Ambulatory Hemodialysis-Technology Landscape and Potential for Patient-Centered Treatment. CJASN , v.15 (1), 2020. pp. 152-159. DOI: 10.2215/CJN.01970219.

HOLDEN, R. J.; CORNET, V. P.; VALDEZ, R. S. Patient ergonomics: 10-year mapping review of patient-centered human factors. Applied Ergonomics, 82, 102972. 2020. DOI:10.1016/j.apergo.2019.102972.

IYER, A., STEIN, L.; FRANKLIN, E. S. Bettering healthcare outcomes through environmental design. Clinical Engineering Handbook, 2020. pp.852-857. DOI:10.1016/b978-0-12-813467-2.00122-x.

INTERNATIONAL ERGONOMICS ASSOCIATION, 2020. Disponível na internet em http:// por < https://www.iea.cc/whats/> Acesso em:25 jan 2019.

JONES. P. H .Design for Care: Innovating Healthcare Experience.New York : Rosenfeld Media.2013.

KARKAR , A.; DAMMANG , M. L.; BOUHAHA. B. M. Stress and burnout among hemodialysis nurses: A single-center, prospective survey study. Saudi Journal of Kidney Diseases and Transplantation. v.26, n.12015. pp.12-18.

KROEMER, K. H. E. Extra-ordinary ergonomics: how to accommodate small and big persons, the disabled and elderly, expectant mothers, and children. FL: Taylor \& Francis Group. 2006.

MACALLISTER, L. Environmental Variables That Influence Patient Satisfaction: a review of the literature. Health Environments Research \& Design JournalAtlanta. 2016. pp.1-15.

MAHNKE, F. H. Color, environment and human response. Canada: John Wiley and Sons Inc.1996.

MATARAZZO, A. K. Z. Composições cromáticas no ambiente hospitalar: estudo de novas abordagens. 2010. Dissertação (Mestrado em Arquitetura e Urbanismo) - Faculdade de Arquitetura e Urbanismo, Universidade de São Paulo.

MELO, G.A.A; AGUIAR, L.L.; SILVA, R.A.; QUIRINO, G.S.; PINHEIRO, A.K.B.;CAETANO, J.A. Factors related to impaired comfort in chronic kidney disease patients on hemodialysis. Rev Bras Enferm. 72(4),. 2019. pp.889-95. DOI:10.1590/0034-71672018-0120.

NEUMANN, W. P.; STEEGE, L. M.; GYUCHAN, T.J.; WIKLUND, M.: Ergonomics and Human Factors in Healthcare System Design - An Introduction to this special issue. IISE Transactions on Occupational Ergonomics and Human Factors, Volume 6, Issue 3-4, 2019. pp.109-115.DOI: 10.1080/24725838.2018.1560927.

RANGEL, M.M. (2011). Cor e ergonomia do ambiente construído: uma investigação da orientação espacial em um ambiente hospitalar. Dissertação (mestrado). Departamento de Artes e Design. Pontificia Universidade Catolica do Rio de Janeiro. 


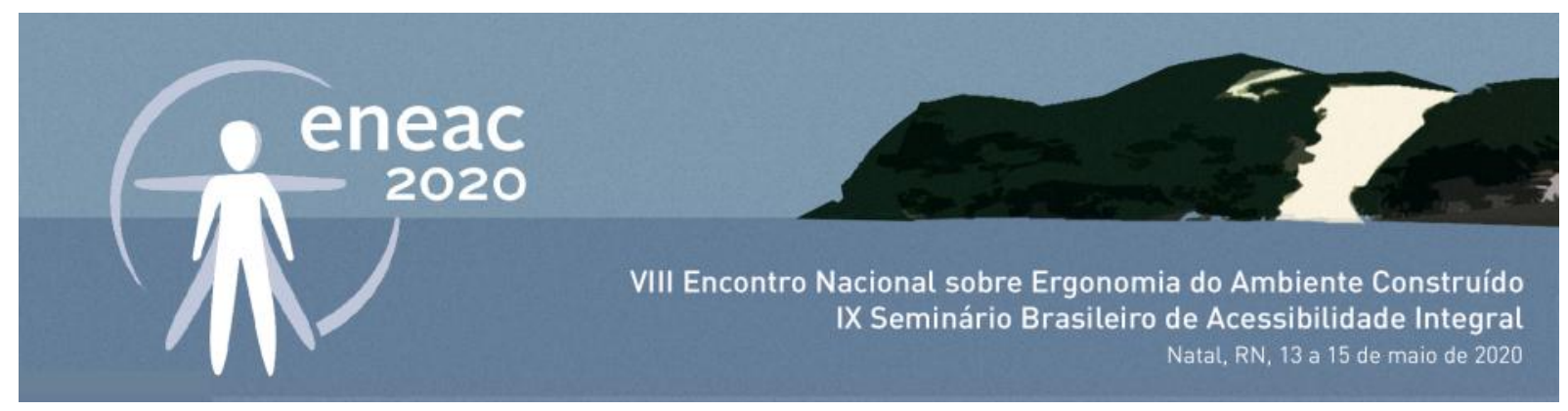

SEO, J. A.; LEE, B. S. Effect of Work Environment on Nursing Performance of Nurses in Hemodialysis Units: Focusing on the Effects of Job Satisfaction and Empowerment. J Korean Acad Nurs Adm. v. 22, n. 2, 2016. pp.178-188.

SERRANHEIRA, F.; UVA, A. S.; SOUSA, P. Ergonomia hospitalar e segurança do doente: mais convergências que divergências. Rev Port Saúde Pública. Vol Temat(10), 2010. pp.58-73.

TOLEDO, C. 3 tendências de tecnologia da informação que deveriam estar no mapa da área de saúde em 2020. Portal Hospitais Brasil.2020. Disponível na internet em http:// por: <portalhospitaisbrasil.com.br/artigo-3-tendencias-detecnologia-da-informacao-que-deveriam-estar-no-mapa-da-area-de-saude-em-2020/> .Acesso em:02 mar 2020.

WISE, B. K.; WISE J.A. The Human Factors of Color in Environmental Design: A Critical Review. Department of Psychology University of Washington. NASA. Document identity: 19890006161 (Acquired Nov 06, 1995), Accession Number: 89N15532, Subject Category: behavioral sciences. Seattle. 1988.

WUNG, S. F.; SCHATZ, M. R. Critical care nurses' cognitive ergonomics related to medical device alarms. Critical Care Nursing Clinics, 30(2), 2018. pp.191-202.

ZUNIIC, A. A new definition of ergonomics. Transations and ergonomics and safety. Volume 1, Issue 1, 2017. pp.1-6. Disponível na internet em http:// por: </ieti.net/TES/>. Acesso em:25 jan 2019. 